\title{
An inter-comparison study to estimate zenith wet delays using VLBI, GPS, and NWP models
}

\author{
Dirk Behrend $^{1}$, Lídia Cucurull ${ }^{1}$, Jordi Vilà ${ }^{2}$ and Rüdiger Haas ${ }^{3}$ \\ ${ }^{1}$ Institut d'Estudis Espacials de Catalunya, Edif. Nexus-204, Gran Capità 2-4, 08034 Barcelona, Spain \\ ${ }^{2}$ Department of Meteorology and Air Quality, Wageningen University, 6701 AP Wageningen, The Netherlands \\ ${ }^{3}$ Onsala Space Observatory, Chalmers University of Technology, SE-439 92 Onsala, Sweden
}

(Received December 20, 1999; Revised June 9, 2000; Accepted June 21, 2000)

\begin{abstract}
Water vapour is a key variable in atmospheric processes and plays a crucial role in atmospheric motions on a wide range of scales in space and time. The water vapour content is approximately proportional to the zenith wet delay (ZWD) which, in turn, constitutes a crucial parameter in geodetic microwave space techniques (VLBI and GPS). Apart from being determined by measurement techniques, the ZWD can also be derived from numerical weather prediction (NWP) models such as the non-hydrostatic MM5 model and the hydrostatic HIRLAM model. At the station Robledo de Chavela (Madrid) ZWD values were derived from VLBI, GPS, MM5, and HIRLAM for the beginning of December 1996. The results of the different techniques agree to the sub-centimetre level with correlation values of 0.87 (GPS vs. MM5), 0.81 (GPS vs. HIRLAM), and 0.84 (MM5 vs. HIRLAM). The correlation VLBI vs. MM5 of 0.78 is based on a short VLBI time series and should be considered preliminary. Further studies with longer time series are necessary to confirm this value. The bias and RMS difference values are all contained in the margin provided by the internal errors.
\end{abstract}

\section{Introduction}

In geodetic space techniques such as the Global Positioning System (GPS) and Very Long Baseline Interferometry (VLBI) the retardation of the microwave signal caused by the presence of water vapour in the atmosphere (troposphere) constitutes a nuisance parameter which is to be removed in order to get a precise location (Tregoning et al., 1998). Thus, the tropospheric delay is usually included in the functional model in form of zenith delay and horizontal delay gradient parameters. Apart from this, it is possible to inject delay and horizontal delay gradient information - that was derived from independent sources - into the GPS or VLBI functional model in order to improve its performance.

Tropospheric delays can also be retrieved from numerical weather prediction (NWP) models. Such models (e.g. MM5 and HIRLAM) are run operationally at national or international weather services and make use of physical models (governing equations), initial atmospheric conditions, and observations (e.g. radiosonde profiles, ground meteorological data) for predicting variables such as temperature, pressure, and humidity.

The inter-comparison of zenith wet delays (ZWDs) resp. precipitable water $(\mathrm{PW})$ derived from different techniques and models provides basic information on the inherent error budget. In previous studies sets of pairs of the aforementioned methods have been analysed with some recent examples being: GPS vs. VLBI (Behrend et al., 1999; Haas et al., 1999), GPS vs. HIRLAM (Yang et al., 1999; Cucurull et

Copy right(C) The Society of Geomagnetism and Earth, Planetary and Space Sciences (SGEPSS); The Seismological Society of Japan; The Volcanological Society of Japan; The Geodetic Society of Japan; The Japanese Society for Planetary Sciences. al., 2000), and GPS vs. MM5 (Cucurull and Vandenberghe, 1999). To date a comparison VLBI vs. NWP as well as an inter-comparison of all three techniques (VLBI, GPS, and NWP models) is not documented in the literature. Thus, we present an investigation of the temporal behaviour of ZWD for a specific site (Robledo de Chavela, Madrid) using all three techniques and including preliminary results of a comparison between VLBI and MM5.

\section{Data Description and Analysis \\ 2.1 GPS data}

In December 1996 (Dec. 3-15) GPS data in a network of 5 stations were acquired in the Madrid Sierra, Spain, employing Trimble 4000SSE GPS receivers at a sampling rate of $30 \mathrm{~s}$ (e.g. Elósegui et al., 1998). The undifferenced dualfrequency carrier-phase and pseudo-range measurements were analysed using the GIPSY (GPS Inferred Positioning SYstem) software package (Webb and Zumberge, 1993). The precise point positioning strategy (Zumberge et al., 1997) yielded estimates of ZWD at the GPS sites with a precision of $5 \mathrm{~mm}$ (Elósegui et al., 1998).

The necessary estimates of the satellite clock corrections and orbits as well as consistent earth-rotation parameters were provided by the IGS (International GPS Service for Geodynamics) and JPL (Jet Propulsion Laboratory). The tropospheric delay was modelled as a random walk $\left(\sigma^{2}=d^{2} \cdot t\right)$ with a drift rate of $d=2.5 \mathrm{~mm} / \sqrt{\mathrm{h}}$. The drift rate for the gradient parameters was $0.3 \mathrm{~mm} / \sqrt{\mathrm{h}}$. We used a cut-off elevation angle of $7^{\circ}$ and an estimation interval of $30 \mathrm{~min}$. For comparison purposes later on, an additional data set was created by averaging the 30 min time series to 3 hour values. In this study we merely utilize the data for the station Robledo 
de Chavela, as it is collocated to a VLBI station.

\subsection{VLBI data}

On December 6, 1996, the European geodetic VLBI experiment EUROPE 6/96 was performed at the Madrid Deep Space Communications Complex (MDSCC) using the DSS65 $34 \mathrm{~m}$ antenna. The nominal observation time of a EUROPE experiment is 24 hours. Unfortunately, Madrid was scheduled only for the second half of this specific experiment due to other commitments leaving an actual observation period of 12 hours. This period was further reduced to 9.5 hours due to bad data at the beginning of the experiment. Thus, EUROPE 6/96 can be considered an uncommon experiment with an unusually short time series. But as there are no other geodetic VLBI sessions at Madrid in the first two weeks of December 1996, it constitutes the only information source available. However, the data are still useful to derive indicative if not significant results.

The VLBI data were analysed using the SOLVE software (Ma et al., 1990). The positions of the radio sources and the station coordinates have been taken from a recent global VLBI solution. Clock parameters were estimated hourly with respect to a reference clock of the European VLBI network. ZWDs as well as horizontal delay gradients were determined using the piecewise linear constrained approach. The estimation interval was chosen to $30 \mathrm{~min}$ for wet delays and 3 hours for gradients. For the upper bound constraints $15 \mathrm{~mm} / \sqrt{\mathrm{h}}$ for the wet delays and $0.82 \mathrm{~mm} / \sqrt{\mathrm{h}}$ for the gradients were taken. The precision (standard deviation) of the ZWD values is in the range of 15 to 20 ps which translates to $4 \ldots 8 \mathrm{~mm}$ in terms of length units.

\subsection{MM5 data}

For the same region and time as the GPS campaign a numerical simulation using the non-hydrostatic Mesoscale Model (MM5) was performed. We set up three (2-way nested) domains with a resolution ranging from $45 \mathrm{~km}$ down to $5 \mathrm{~km}$. Gridded precipitable water (PW) values were simulated in the finest domain. The grid values were calculated by integrating the specific humidity in the vertical, in this case over 23 vertical layers. In order to estimate point values for the VLBI and GPS sites (in the horizontal) the grid values were bilinearly interpolated from the four closest grid points. The individual PW values were averaged over $30 \mathrm{~min}$ intervals to obtain values that are coherently comparable with the GPS and VLBI results. The precision of the PW is about $2 \mathrm{~mm}$ (Cucurull and Vandenberghe, 1999). As was done with the GPS data, the $30 \mathrm{~min}$ time series was averaged to 3 hour values. Finally, the PW were converted to ZWD values using the relationship $\mathrm{PW}=\Pi \cdot \mathrm{ZWD}$ (e.g. Tregoning et al., 1998 ) where the dimensionless conversion factor was chosen to $\Pi \approx 0.15$ (Emardson and Derks, 1999).

\subsection{HIRLAM data}

The HIRLAM (HIgh Resolution Limited Area Modelling) short-range NWP model is a complete analysis and forecast system over a limited area. It is the operational model at the Spanish Weather Service (INM) and is routinely run with two horizontal resolutions, $0.5^{\circ} \times 0.5^{\circ}$ and $0.2^{\circ} \times 0.2^{\circ}$, and 31 vertical levels. The forecast model is hydrostatic with Eulerian grid-point numbers and provides time series of 3 hours. The accuracy of HIRLAM's predictions of pressure fields is better than $1.5 \mathrm{hPa}$, and of the order of $15 \mathrm{~mm}$ for
ZWD (Cucurull et al., 2000).

For obtaining ZWDs two different approaches were followed: (1) calculation of PW by integrating the specific humidity in the vertical followed by the conversion of the PW to ZWD like in the MM5 case; (2) generation of refractivity profiles from HIRLAM's temperature, pressure, and humidity profiles followed by integration of the refractivity along the zenith direction yielding directly ZWD. The difference between the two procedures is negligible (less than $0.5 \mathrm{~mm}$ in PW) which may be considered a verification of the chosen conversion factor $\Pi$. To estimate point values for the VLBI and GPS sites again bilinear interpolation from the four closest grid points was used. A thorough description of the HIRLAM analysis is given in Cucurull et al. (2000).

\section{Comparison and Results}

From the description of the data analysis in Section 2 the precision of the four techniques can be compiled (cf. Table 1). The measurement techniques provide ZWDs two to three times higher in precision than the retrievals from the NWP models. For improving the models in a data assimilation context the PW values should have accuracies of $2 \mathrm{~mm}$ or better (Cucurull et al., 2000).

Table 1. Precision of ZWD and PW derived from geodetic space techniques (GPS, VLBI) and NWP models (MM5, HIRLAM).

\begin{tabular}{lcc}
\hline Method & ZWD & PW \\
\hline GPS & $\sim 5 \mathrm{~mm}$ & $\sim 1 \mathrm{~mm}$ \\
VLBI & $\sim 4 \ldots 8 \mathrm{~mm}$ & $\sim 1 \ldots 2 \mathrm{~mm}$ \\
MM5 & $\sim 15 \mathrm{~mm}$ & $\sim 2 \mathrm{~mm}$ \\
HIRLAM & $\sim 15 \mathrm{~mm}$ & $\sim 2 \mathrm{~mm}$ \\
\hline
\end{tabular}

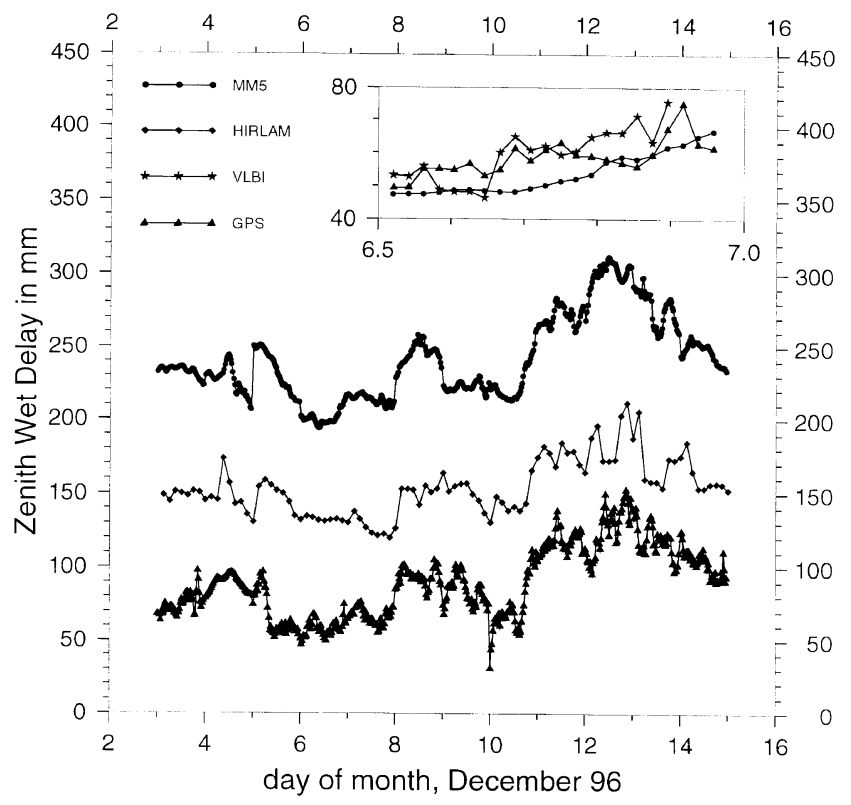

Fig. 1. Time series of ZWD values (MM5, HIRLAM, GPS) at Robledo de Chavela. MM5 and HIRLAM are offset by $150 \mathrm{~mm}$ resp. $75 \mathrm{~mm}$ for mapping purposes. Inlay: ZWDs derived from VLBI, GPS, and MM5 for the length of the VLBI experiment. 
Table 2. Comparison of GPS, VLBI, MM5, and HIRLAM derived ZWD values. Unit of bias and RMS difference: mm.

\begin{tabular}{lcccccc}
\hline Meth. 1 & Meth. 2 & \#points & Resolution & Correlation & Bias & RMS diff. \\
\hline GPS & MM5 & 564 & $30 \mathrm{~min}$ & 0.874 & 2.23 & 14.09 \\
GPS & MM5 & 95 & $3 \mathrm{~h}$ & 0.864 & 2.38 & 14.48 \\
GPS & MM5 & 19 & $30 \mathrm{~min}$ & 0.550 & -5.31 & 4.23 \\
GPS & HIRLAM & 95 & $3 \mathrm{~h}$ & 0.812 & -9.97 & 14.40 \\
VLBI & MM5 & 19 & $30 \mathrm{~min}$ & 0.781 & -7.52 & 5.20 \\
\hline VLBI & GPS & 19 & $30 \mathrm{~min}$ & 0.654 & -2.21 & 6.10 \\
MM5 & HIRLAM & 95 & $3 \mathrm{~h}$ & 0.835 & -12.35 & 16.51 \\
\hline
\end{tabular}

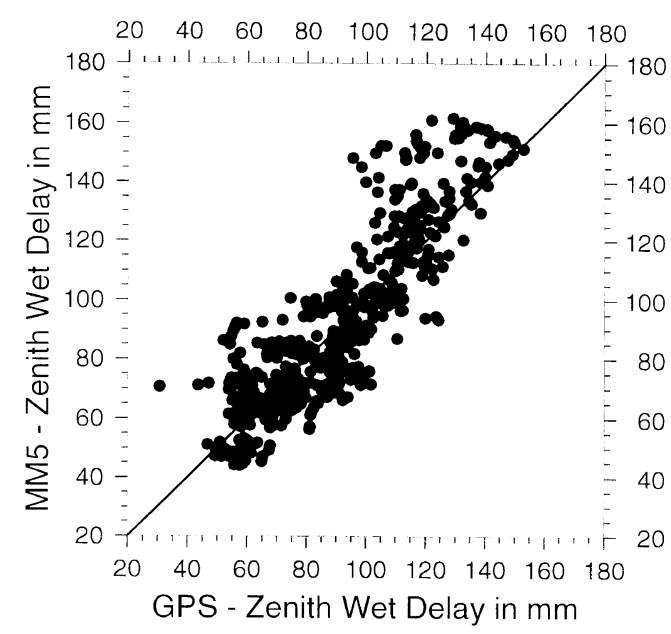

Fig. 2. Scatter plot for Robledo using GPS and MM5 derived ZWD values. Dec. 3-15, 1996. 30 min sampling.

Figure 1 shows the time series of the ZWD values for the station Robledo de Chavela for the period Dec. 3-15, 1996. The variation of the ZWD between $30 \mathrm{~mm}$ and $160 \mathrm{~mm}$ can mainly be attributed to the passage of two frontal systems (Dec. 4-5 and 12-14). These winter frontal systems are identifiable by initial increases in the atmospheric moisture followed by decreases after the passage of the front. The agreement between the independent ZWD profiles is reasonably well and shall be quantified by computation of correlation as well as bias and RMS difference values between sets of pairs of the involved techniques (correlation analysis). The rapid increase on day 5 could be associated with fluctuations in the water vapour fields due to the passage of the first front.

The results of the correlation analysis are summarized in Table 2 and are depicted exemplarily in the scatter plots of Figs. 2 to 4. The GPS vs. MM5 comparison was done with two time resolutions ( $30 \mathrm{~min}$ and $3 \mathrm{~h}$ ) and two lengths for the time series ( 2 weeks and $9.5 \mathrm{~h}$ ). Lowering the time resolution from $30 \mathrm{~min}$ to $3 \mathrm{~h}$ has no significant effect, as correlation, bias, and RMS difference stay almost the same. In contrast to this, the confinement of the time span to the length of the VLBI experiment $(9.5 \mathrm{~h})$ causes a decrease of the RMS difference value (as was to be expected due to the lesser num-

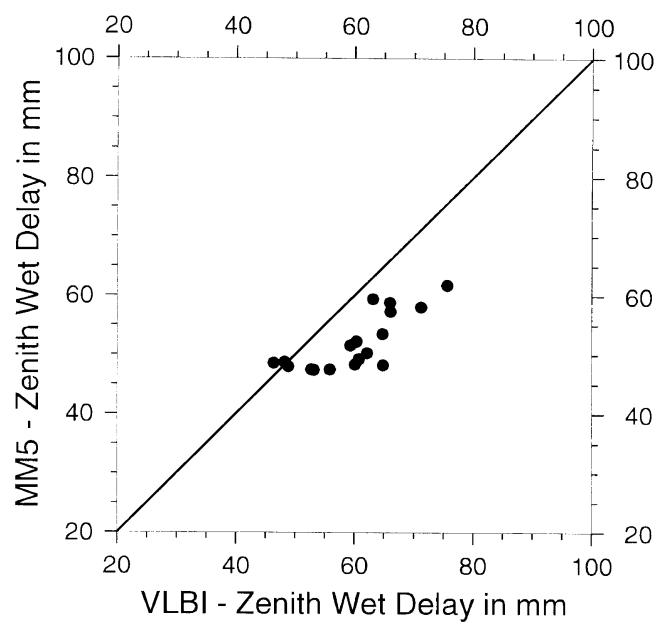

Fig. 3. Scatter plot for Robledo using VLBI and MM5 derived ZWD values Dec. 6, 1996 (9.5 h). 30 min sampling.

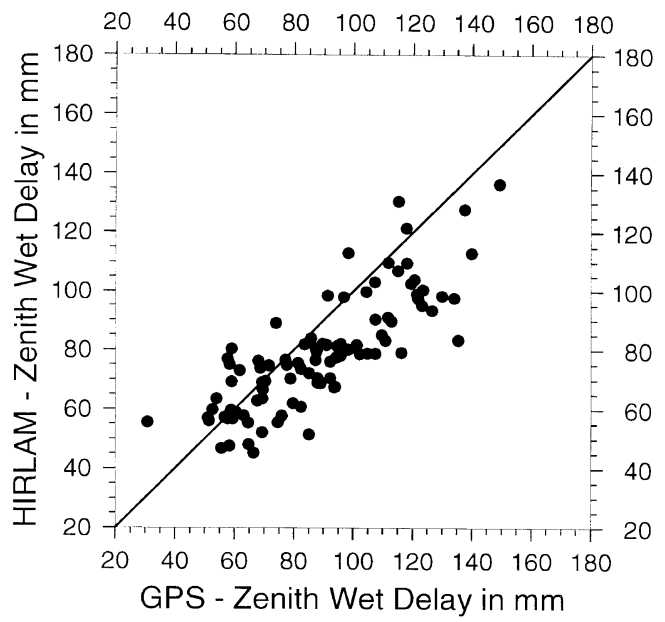

Fig. 4. Scatter plot for Robledo using GPS and HIRLAM derived ZWD values. Dec. 3-15, 1996. 3 h sampling.

ber of input values), a reduction of the correlation coefficient, and a change of the bias value from $2.23 \mathrm{~mm}$ to $-5.31 \mathrm{~mm}$. The latter may be attributed to the fact that the bias between GPS and MM5 is not constant, but depends on the degree of 
moisture: MM5 overestimates the water vapour content for high moisture values and underestimates it for low moisture values (see Figs. 1 and 2).

The time series of the VLBI data is too short in order to give significant results regarding systematic errors of VLBI with respect to the other methods. But since the VLBI vs. GPS comparisons obtained with the short data set (cf. Table 2) agree reasonably well with results reported in Haas et al. (1999), Gradinarsky et al. (1999), Behrend et al. (1999), and Gradinarsky et al. (2000), the comparison results may at least be considered indicative. The VLBI experiment was run in a period of low moisture during which MM5 tends to underestimate the ZWDs (see above). When comparing the VLBI-derived with the MM5-derived ZWD values (Table 2 and Fig. 3) the RMS difference is slightly higher than in the GPS vs. MM5 case. This holds true also for the bias values, where the increase is equal to the bias VLBI vs. GPS. The correlation value of 0.78 is significantly higher than the GPS vs. MM5 (short time series) one suggesting a better agreement between VLBI and MM5 than between GPS and MM5 for this special case. With sufficiently long time series (e.g. two weeks) correlation values on the order of the GPS vs. MM5 results may be expected. This is to be verified by further studies using more data material, but the initial results are promising.

As the HIRLAM-derived ZWDs are available at a sampling rate of 3 hours, the ZWDs are only compared with the GPS-derived and the MM5-derived ZWDs over the two weeks of the GPS campaign (cf. Table 2 and Fig. 4). The correlation values are in the $0.80 \ldots 0.85$ range. The biases are somewhat larger than the previous ones with values of about $10 \ldots 12 \mathrm{~mm}$ (HIRLAM lower than GPS and MM5) indicating that HIRLAM underestimates the moisture content of the troposphere as compared to GPS and MM5. The RMS difference is around $15 \mathrm{~mm}$ corresponding to the error margin given by the formal errors of each technique. This corroborates to a certain extent the validity of the precision values compiled in Table 1.

\section{Conclusions}

We inter-compared ZWD values derived from GPS, VLBI, MM5, and HIRLAM data for the station Robledo de Chavela (Madrid), Spain. The results agree to the sub-centimetre level. The correlation values obtained from a time series of two weeks amount to 0.87 (GPS vs. MM5), 0.81 (GPS vs. HIRLAM), and 0.84 (MM5 vs. HIRLAM); the bias and RMS difference values fall within the error frames provided by the internal accuracies of the respective methods. The VLBI data employed in the comparison cover a time span of 9.5 hours, so that the comparison results should be considered indicative only. With a correlation value of 0.78 (VLBI vs. MM5) they, nevertheless, look promising.

The geodetic space techniques (VLBI and GPS) provide estimates of the ZWD at the $5 \mathrm{~mm}$ level, whereas the NWP models (MM5 and HIRLAM) give ZWD values with an accuracy of about $15 \mathrm{~mm}$. This means that the geodetic space techniques furnish ZWD estimates superior to the NWPderived values by a factor of about 3. Thus, both VLBI and GPS can be employed to validate NWP models and may be utilized as additional information source for the models in a data assimilation context. Due to the favourable spatial distribution of GPS stations, the low operational costs (as compared to VLBI) and the near-real-time availability, GPS constitutes the obvious choice for improving NWP models. VLBI, on the other hand, lends itself to independent checks at selected sites.

Acknowledgments. Dirk Behrend and Rüdiger Haas are supported by the European Union within the TMR programme under contract FMRX-CT960071.

\section{References}

Behrend, D., A. Rius, R. Haas, L. P. Gradinarsky, J. M. Johansson, and S. J. Keihm, Comparison of independently derived atmospheric parameters, IUGG XXII General Assembly, Birmingham, England, 1999.

Cucurull, L. and F. Vandenberghe, Comparison of PW estimated from MM5 and GPS data, MM5 Workshop '99, Boulder, Colorado, U.S.A., 1999.

Cucurull, L., B. Navascues, G. Ruffini, P. Elósegui, A. Rius, and J. Vilà, On the use of GPS to validate NWP systems: the HIRLAM model, J. Atmos. and Ocean. Tech., 17(6), 773-787, 2000.

Elósegui, P., A. Rius, J. L. Davis, G. Ruffini, S. J. Keihm, B. Bürki, and L. P. Kruse, An experiment for estimation of the spatial and temporal variations of water vapor using GPS data, Phys. Chem. Earth, 23(1), 125-130, 1998.

Emardson, T. R. and H. J. P. Derks, On the relation between the wet delay and the integrated precipitable water vapor in the European atmosphere, Meteorol. Appl., 6, 1-12, 1999.

Gradinarsky, L. P., R. Haas, J. M. Johansson, and G. Elgered, Comparison of atmospheric parameters estimated from VLBI, GPS and microwave radiometer data, Proc. 13th Work. Meet. Europ. VLBI f. Geod. Astrom., 161-165, Viechtach, 1999.

Gradinarsky, L. P., R. Haas, G. Elgered, and J. M. Johansson, Wet path delay and delay gradients inferred from microwave radiometer, GPS and VLBI observations, Earth Planets Space, 52, this issue, 695-698, 2000.

Haas, R., L. P. Gradinarsky, J. M. Johansson, and G. Elgered, The atmospheric propagation delay: A common error source for colocated space techniques of VLBI and GPS, Proc. Int. Work. "Geod. Meas. Coll. Space Tech. Earth" (GEMSTONE), 230-234, Koganei, Tokyo, 1999.

Ma, C., J. M. Sauber, L. J. Bell, T. A. Clark, D. Gordon, and W. E. Himwich, Measurement of horizontal motions in Alaska using very long baseline interferometry, J. Geophys. Res., 95(B13), 21991-22011, 1990.

Tregoning, P., R. Boers, D. O'Brien, and M. Hendy, Accuracy of absolute precipitable water vapor estimates from GPS observations, J. Geophys. Res., 103(D22), 28701-28710, 1998.

Webb, F. H. and J. F. Zumberge, An introduction to GIPSY/OASIS-II, JPL Publication, D-11088, 300 pp., 1993.

Yang, X., B. H. Sass, G. Elgered, J. M. Johansson, and T. R. Emardson, A comparison of precipitable water vapor estimates by an NWP simulation and GPS observations, J. Appl. Meteorol., 38, 941-956, 1999.

Zumberge, J. F., M. B. Heflin, D. C. Jefferson, M. M. Watkins, and F. H. Webb, Precise point positioning for the efficient and robust analysis of GPS data from large networks, Geophys. Res. Lett., 102, 5005-5017, 1997.

D. Behrend (e-mail: behrend@ieec.fcr.es), L. Cucurull, J. Vilà, and R. Haas 